\title{
HIGHER DERIVATIONS ON RINGS AND MODULES
}

\author{
PAUL E. BLAND
}

Received 27 June 2001 and in revised form 14 July 2005

Let $\tau$ be a hereditary torsion theory on $\operatorname{Mod}_{R}$ and suppose that $Q_{\tau}: \operatorname{Mod}_{R} \rightarrow \operatorname{Mod}_{R}$ is the localization functor. It is shown that for all $R$-modules $M$, every higher derivation defined on $M$ can be extended uniquely to a higher derivation defined on $Q_{\tau}(M)$ if and only if $\tau$ is a higher differential torsion theory. It is also shown that if $\tau$ is a TTF theory and $C_{\tau}: M \rightarrow M$ is the colocalization functor, then a higher derivation defined on $M$ can be lifted uniquely to a higher derivation defined on $C_{\tau}(M)$.

\section{Introduction}

Rim has shown in [16] that under certain conditions a higher antiderivation $d: M \rightarrow M$ can be extended to a higher antiderivation $d^{\tau}: Q_{\tau}(M) \rightarrow Q_{\tau}(M)$, where $Q_{\tau}: \operatorname{Mod}_{R} \rightarrow$ $\operatorname{Mod}_{R}$ is the localization functor [10] at a hereditary torsion theory $\tau$ on $\operatorname{Mod}_{R}$. By selecting the involution on the ring in the definition of a higher antiderivation to be the identity mapping on $R$, a higher antiderivation $d: M \rightarrow M$ becomes a higher derivation as defined by Ribenboim in [15]. Thus Rim's results, which generalize the results of Golan [9], show that a higher derivation $d: M \rightarrow M$ can be extended to a higher derivation $d^{\tau}: Q_{\tau}(M) \rightarrow Q_{\tau}(M)$ whenever the conditions of his proposition are met. Uniqueness of extensions of higher (anti-) derivations and the necessary and sufficient conditions for the existence of these extensions were not addressed. The purpose of this paper is to introduce higher differential torsion theories and to show that a higher derivation $d: M \rightarrow M$ can always be extended uniquely to $Q_{\tau}(M)$, the module of quotients of $M$, if and only if $\tau$ is a higher differential torsion theory on $\operatorname{Mod}_{R}$. We also show that a higher derivation $d: M \rightarrow M$ can be lifted uniquely to the module of coquotients $C_{\tau}(M)$ of $M$ at a TTF theory on $\operatorname{Mod}_{R}$.

Throughout this paper $R$ will be an associative ring with identity $1, \operatorname{Mod}_{R}$ will denote the category of unitary right $R$-modules and all modules and module homomorphisms will be in $\operatorname{Mod}_{R}$. 


\section{Differential torsion theory}

A torsion theory $\tau$ on $\operatorname{Mod}_{R}$ is a pair $(\mathrm{T}, \mathrm{F})$ of classes of $R$-modules such that the following conditions hold.

(1) $\mathrm{T} \cap \mathrm{F}=0$.

(2) If $M \rightarrow N \rightarrow 0$ is an exact sequence in $\operatorname{Mod}_{R}$ and $M \in \mathrm{T}$, then $N \in \mathrm{T}$.

(3) If $0 \rightarrow M \rightarrow N$ is an exact sequence in $\operatorname{Mod}_{R}$ and $N \in \mathrm{F}$, then $M \in \mathrm{F}$.

(4) For each $R$-module $M$, there is a short exact sequence $0 \rightarrow T \rightarrow M \rightarrow F \rightarrow 0$ in $\operatorname{Mod}_{R}$ with $T \in \mathrm{T}$ and $F \in \mathrm{F}$.

It follows that the class $\mathrm{T}$ is closed under factor modules, direct sums, and extensions, and that $\mathrm{F}$ is closed under submodules, direct products, and extensions. A class $\mathrm{C}$ of $R$ modules is said to be closed under extensions if whenever $0 \rightarrow M_{1} \rightarrow M \rightarrow M_{2} \rightarrow 0$ is a short exact sequence in $\operatorname{Mod}_{R}$ and $M_{1}$ and $M_{2}$ are in $\mathrm{C}$, then $M$ is in C. Modules in T will be called $\tau$-torsion and those in $\mathrm{F}$ are called $\tau$-torsion-free. Each $R$-module $M$ has a largest and necessarily unique $\tau$-torsion submodule given by $t_{\tau}(M)=\sum_{N \in S} N$, where $S$ is the set of $\tau$-torsion submodules of $M$. A torsion theory will be called hereditary if $\mathrm{T}$ is closed under submodules and it will be called cohereditary if $\mathrm{F}$ is closed under factor modules. Standard results and terminology on torsion theory can be found in $[4,10]$ while general information on rings and modules can be found in [2]. Finally, if $N$ is a submodule of an $R$-module $M$, then for any $x \in M,(N: x)$ will denote the right ideal of $R$ given by $\{a \in R \mid x a \in N\}$ and $(0: x)$ is the right ideal $\{a \in R \mid x a=0\}$.

A nonempty collection $\mathscr{F}$ of right ideals of $R$ is said to be a (Gabriel) filter [8] if the following two conditions hold.

(1) If $K \in \mathscr{F}$, then $(K: a) \in \mathscr{F}$ for each $a \in R$.

(2) If $I$ is a right ideal of $R$ and $K \in \mathscr{F}$ is such that $(I: a) \in \mathscr{F}$ for each $a \in K$, then $I \in \mathscr{F}$.

It can be shown that each filter of right ideals of $R$ also satisfies the following three conditions.

(3) If $J \in \mathscr{F}$ and $K$ is a right ideal of $R$ such that $J \subseteq K$, then $K \in \mathscr{F}$.

(4) If $J, K \in \mathscr{F}$, then $J \cap K \in \mathscr{F}$.

(5) If $J, K \in \mathscr{F}$, then $J K \in \mathscr{F}$.

If $\tau$ is a hereditary torsion theory on $\operatorname{Mod}_{R}$, then $\mathscr{F}_{\tau}=\{K \mid K$ is a right ideal of $R$ and $R / K \in \mathrm{T}\}$ is a filter. An element $x$ of an $R$-module $M$ is said to be a $\tau$-torsion element of $M$ if there is a $K \in \mathscr{F}_{\tau}$ such that $x K=0$. The set of all $\tau$-torsion elements of $M$ is the $\tau$ torsion submodule $t_{\tau}(M)$ of $M$ mentioned earlier. Moreover, an $R$-module $M$ is $\tau$-torsion if $t_{\tau}(M)=M$ and $\tau$-torsion-free if $t_{\tau}(M)=0$. Conversely, if $\mathscr{F}$ is a filter of right ideals of $R$ and $t(M)=\{x \in M \mid x K=0$ for some $K \in \mathscr{F}\}$, then $\tau=(\mathrm{T}, \mathrm{F})$ is a hereditary torsion theory on $\operatorname{Mod}_{R}$, where $\mathrm{T}=\{M \mid t(M)=M\}$ and $\mathrm{F}=\{M \mid t(M)=0\}$. It follows that there is a one-to-one correspondence between hereditary torsion theories on $\operatorname{Mod}_{R}$ and filters of right ideals of $R$.

An additive mapping $\delta: R \rightarrow R$ such that $\delta(a b)=\delta(a) b+a \delta(b)$ for all $a, b \in R$ is said to be a derivation on $R$ and, given a derivation $\delta$ on $R$, an additive mapping $d: M \rightarrow M$ such that $d(x a)=d(x) a+x \delta(a)$ is a derivation on $M$. Important to our discussion is the concept of a differential torsion theory, introduced in [5]. If $\delta$ is a derivation on $R$ and $\mathscr{F}$ is a filter of right ideals of $R$, then $\mathscr{F}$ is called a differential filter (with respect to $\delta$ ) if 
for each $K \in \mathscr{F}$ there is an $I \in \mathscr{F}$ such that $\delta(I) \subseteq K$. If $\tau$ is a hereditary torsion theory on $\operatorname{Mod}_{R}$ and the corresponding filter $\mathscr{F}_{\tau}$ is a differential filter, then $\tau$ is said to be a differential torsion theory.

Remark 2.1. If $\mathscr{F}$ is a differential filter and $K \in \mathscr{F}$, then there is an $I \in \mathscr{F}$ such that $\delta(I) \subseteq$ $K$. The right ideal $I$ can actually be chosen so that $I \subseteq K$. Indeed, if $I \in \mathscr{F}$ is such that $\delta(I) \subseteq K$ and $I^{\prime}=I \cap K$, then $I^{\prime} \in \mathscr{F}, I^{\prime} \subseteq K$, and $\delta\left(I^{\prime}\right) \subseteq K$.

The following example shows that differential torsion theories do indeed exist. We will see additional examples later.

Example 2.2. If $S$ is a multiplicatively closed set of elements of $R$ that is a right denominator set [12], then $S$ satisfies the following conditions.

(1) If $(a, s) \in R \times S$, then there is a $(b, t) \in R \times S$ such that $a t=s b$.

(2) If $s a=0$ with $s \in S$ and $a \in R$, then $a t=0$ for some $t \in S$.

If $\delta$ is a derivation on $R$, then the set $\mathscr{F}=\{K \mid K$ is a right ideal of $R$ and $K \cap S \neq \varnothing\}$ is a filter of right ideals of $R$. If $K \in \mathscr{F}$, let $s \in K \cap S$. Since $(\delta(s), s) \in R \times S$, there is a $(b, t) \in R \times S$ such that $\delta(s) t=s b$. Now $\delta(s t)=\delta(s) t+s \delta(t)=s b+s \delta(t) \in s R \subseteq K$, so if $a \in R$, then $\delta(s t a)=\delta(s t) a+s t \delta(a) \in K$. Hence $\delta(s t R) \subseteq K$. Therefore $\mathscr{F}_{F}$ is a differential filter, so the torsion theory determined by $\mathscr{F}$ is a differential torsion theory.

The following lemma gives two conditions that characterize differential filters.

Lemma 2.3. Let $\tau$ be a hereditary torsion theory on $\operatorname{Mod}_{R}$ with corresponding filter $\mathscr{F}_{\tau}$. Then the following are equivalent for a derivation $\delta$ on $R$.

(1) $\mathscr{F}_{\tau}$ is a differential filter.

(2) For every $R$-module $M$, if $x \in t_{\tau}(M)$, then there is an $I \in \mathscr{F}_{\tau}$ such that $\delta(I) \subseteq(0: x)$.

(3) For every $R$-module $M$, if $d: M \rightarrow M$ is a derivation on $M$, then $d\left(t_{\tau}(M)\right) \subseteq t_{\tau}(M)$.

Proof. $(1) \Rightarrow(3)$. If $x \in t_{\tau}(M)$, then there is a $K \in \mathscr{F}_{\tau}$ such that $x K=0$ and an $I \in \mathscr{F}_{\tau}$ such that $\delta(I) \subseteq K$. Thus if $a \in I \cap K \in \mathscr{F}_{\tau}$, then we see that $0=d(x a)=d(x) a+x \delta(a)=$ $d(x) a$. Hence $d(x)(I \cap K)=0$, so $d(x) \in t_{\tau}(M)$.

(3) $\Rightarrow(2)$. If $x \in t_{\tau}(M)$ and $a \in R$, then $d(x)$ and $d(x a)$ are in $t_{\tau}(M)$. Thus $(0: d(x)) \cap$ $(0: d(x a)) \in \mathscr{F}_{\tau}$. Therefore $I=(0: d(x)) \cap(0: d(x a)) \in \mathscr{F}_{\tau}$. If $a \in I$, then $d(x) a=$ $d(x a)=0$, so $0=d(x a)=d(x) a+x \delta(a)=x \delta(a)$. Thus $\delta(a) \in(0: x)$ and we have $\delta(I) \subseteq$ $(0: x)$.

$(2) \Rightarrow(1)$. If $K \in \mathscr{F}_{\tau}$, then we need to find an $I \in \mathscr{F}_{\tau}$ such that $\delta(I) \subseteq K$. Since $R / K$ is $\tau$-torsion, $1+K \in t_{\tau}(R / K)$, so by (2) there is an $I \in \mathscr{F}_{\tau}$ such that $\delta(I) \subseteq(0: 1+K)=K$.

\section{Higher differential torsion theory}

Let $n$ be a nonnegative integer. Then a family of additive mapping $\delta=\left\{\delta_{i}: R \rightarrow R\right\}_{i=0}^{n}$, denoted by $\delta: R \rightarrow R$, is said to be a higher derivation (on $R$ ) of order $n$ provided that

$$
\begin{aligned}
\delta_{i}(a b) & =\delta_{i}(a) \delta_{0}(b)+\delta_{i-1}(a) \delta_{1}(b)+\cdots+\delta_{0}(a) \delta_{i}(b) \\
& =\delta_{i}(a) b+\delta_{i-1}(a) \delta_{1}(b)+\cdots+a \delta_{i}(b)
\end{aligned}
$$


for all $a, b \in R$ and $i=0,1, \ldots, n$, where it is understood that $\delta_{0}$ is the identity mapping on $R$. If $\delta: R \rightarrow R$ is a higher derivation of order 1 , then $\delta_{1}(a b)=\delta_{1}(a) b+a \delta_{1}(b)$ for all $a, b \in$ $R$, so a higher derivation on $R$ of order 1 gives a derivation on $R$. Given a higher derivation $\delta: R \rightarrow R$ of order $n$, a family of additive mappings $d=\left\{d_{i}: M \rightarrow M\right\}_{i=0}^{n}$, denoted by $d: M \rightarrow M$, is a higher derivation (on $M$ ) of order $n$ if for $x \in M$ and $a \in R$

$$
\begin{aligned}
d_{i}(x a) & =d_{i}(x) \delta_{0}(a)+d_{i-1}(x) \delta_{1}(a)+\cdots+d_{0}(x) \delta_{i}(a) \\
& =d_{i}(x) a+d_{i-1}(x) \delta_{1}(a)+\cdots+x \delta_{i}(a)
\end{aligned}
$$

for $i=0,1, \ldots, n$ with the understanding that $d_{0}$ will always be the identity mapping on $M$. A higher derivation $d: M \rightarrow M$ of order 1 gives $d_{1}(x a)=d_{1}(x) a+x \delta_{1}(a)$, so $d$ produces a derivation on $M$. To simplify terminology, a higher derivation of order $n$ will be referred to simply as a derivation of order $n$.

Remark 3.1. If $\delta: R \rightarrow R$ and $d: M \rightarrow M$ are derivations of order $n$ and $0 \leq k \leq n$, then $\bar{\delta}=$ $\left\{\delta_{i}: R \rightarrow R\right\}_{i=0}^{k}$ and $\bar{d}=\left\{d_{i}: M \rightarrow M\right\}_{i=0}^{k}$ are derivations of order $k$, where $\bar{d}$ is taken with respect to $\bar{\delta}$. This will subsequently be described by saying that $d$ produces derivations of order $k$ for $k=0,1, \ldots, n$.

Let $\mathscr{F}$ be the filter of right ideals of $R$ and suppose that $\delta: R \rightarrow R$ is derivation of order $n$. If $\mathscr{F}$ is such that for each $K \in \mathscr{F}$ there is an $I \in \mathscr{F}$ such that $\delta_{i}(I) \subseteq K$ for $i=0,1, \ldots, n$, then we will say that $\mathscr{F}$ is a differential filter of order $n$. In this setting, the corresponding hereditary torsion theory $\tau$ is called a differential torsion theory of order $n$. We now fix the derivation $\delta: R \rightarrow R$ of order $n$ and assume, unless stated otherwise, that every derivation $d: M \rightarrow M$ of order $n$, every differential filter $\mathscr{F}$ of order $n$, and every differential torsion theory $\tau$ of order $n$ is taken with respect to $\delta$. Because of Remark 3.1 every differential filter (differential torsion theory) of order $n$ produces a differential filter (differential torsion theory) of order $k$ for $k=0,1, \ldots, n$.

In the previous section an example of a differential torsion theory was given. Differential torsion theories of order $n$ can also be shown to exist. In each of the following examples $\delta$ is a derivation on $R$ of order $n$. In particular, we also see that each of the following is also a differential torsion theory.

Example 3.2. Let $R$ be a commutative ring and suppose that $\mathscr{F}_{F}$ is a filter of ideals of $R$. If $I \in \mathscr{F}$, then $I^{2} \in \mathscr{F}$, and it follows that $\delta_{0}\left(I^{2}\right)=I^{2} \subseteq I$. So suppose that $\delta_{i}\left(I^{2}\right) \subseteq I$ for all $i$ with $0 \leq i<n$. If $a, b \in I$, then

$$
\delta_{n}(a b)=\delta_{n}(a) b+\delta_{n-1}(a) \delta_{1}(b)+\cdots+\delta_{1}(a) \delta_{n-1}(b)+a \delta_{n}(b)
$$

which is easily seen to be in $I$. Since $\delta_{n}$ is additive, we have that $\delta_{n}\left(I^{2}\right) \subseteq I$. Therefore $\mathscr{F}_{\tau}$ is a differential filter of order $n$, so the corresponding hereditary torsion theory on $\operatorname{Mod}_{R}$ is a differential torsion theory of order $n$. Thus for a commutative ring every hereditary torsion theory on $\operatorname{Mod}_{R}$ is a differential torsion theory of order $n$.

Example 3.3. Jans has shown in [11] that if $\tau=(\mathrm{T}, \mathrm{F})$ is a hereditary torsion on $\operatorname{Mod}_{R}$ such that $\mathrm{T}$ is closed under direct products, then there is an idempotent ideal $I \in \mathscr{F}_{\tau}$ such 
that $I \subseteq K$ for each $K \in \mathscr{F}_{\tau}$. Using the same procedure as in the previous example, we can show that $\delta_{i}(I) \subseteq K$ for $i=0,1, \ldots, n$. Thus $\tau$ is a differential torsion theory of order $n$.

Example 3.4. If $R$ is left perfect, then Alin and Armendariz [1] and Dlab [7] have independently proved that if $\tau=(\mathrm{T}, \mathrm{F})$ is a hereditary torsion theory on $\operatorname{Mod}_{R}$, then $\mathrm{T}$ is closed under direct products. Thus, we see from the previous example that if $R$ is left perfect, then every hereditary torsion theory on $\operatorname{Mod}_{R}$ is a differential torsion theory of order $n$.

With the definitions of a differential filter of order $n$ and a differential torsion theory of order $n$ in place, one might expect that Lemma 2.3 can be generalized to higher differential filters. The following lemma shows that this is indeed the case.

Lemma 3.5. If $\tau$ is a hereditary torsion theory on $\operatorname{Mod}_{R}$ with corresponding filter $\mathscr{F}_{\tau}$, then the following are equivalent for an integer $n \geq 0$.

(1) $\mathscr{F}_{\tau}$ is a differential filter of order $n$.

(2) For every $R$-module $M$ and each derivation $d: M \rightarrow M$ of order $n$, if $x \in t_{\tau}(M)$, then there is an $I \in \mathscr{F}_{\tau}$ such that $\delta_{i}(I) \subseteq\left(0: d_{n-i}(x)\right)$ for $i=0,1, \ldots, n$.

(3) For every $R$-module $M$, if $d: M \rightarrow M$ is a derivation of order $n$, then $d_{i}\left(t_{\tau}(M)\right) \subseteq$ $t_{\tau}(M)$ for $i=0,1, \ldots, n$.

Proof. (1) $\Rightarrow(3)$. If $n=0$, then it is trivial that (1) $\Rightarrow(3)$, so suppose (1) $\Rightarrow(3)$ for every integer $k, 0 \leq k<n$. Now let $\mathscr{F}_{\tau}$ be a differential filter of order $n$ and suppose that $d: M \rightarrow$ $M$ is a derivation of order $n$. In view of Remark 3.1, $d$ produces a derivation $\left\{d_{i}: M \rightarrow\right.$ $M\}_{i=0}^{n-1}$ on $M$ of order $n-1$ and it follows that $\mathscr{F}_{\tau}$ is a differential filter of order $n-1$. So if $x \in t_{\tau}(M)$, then the induction hypothesis shows that $d_{1}(x), \ldots, d_{n-1}(x) \in t_{\tau}(M)$. Hence each of

$$
(0: x),\left(0: d_{1}(x)\right),\left(0: d_{2}(x)\right), \ldots,\left(0: d_{n-1}(x)\right)
$$

is in $\mathscr{F}_{\tau}$. Since $\mathscr{F}_{\tau}$ is a filter, $K=\cap_{i=0}^{n-1}\left(0: d_{i}(x)\right) \in \mathscr{F}_{\tau}$, so since $\mathscr{F}_{\tau}$ is a differential filter of order $n$, there is an $I \in \mathscr{F}_{\tau}$ such that $\delta_{i}(I) \subseteq K$ for $i=0,1, \ldots, n$. If $a \in I$, then

$$
d_{n}(x a)=d_{n-1}(x) \delta_{1}(a)=\cdots=d_{1}(x) \delta_{n-1}(a)=x \delta_{n}(a)=0,
$$

SO

$$
d_{n}(x a)=d_{n}(x) a+d_{n-1}(x) \delta_{1}(a)+\cdots+d_{1}(x) \delta_{n-1}(a)+x \delta_{n}(a)
$$

gives $d_{n}(x) a=0$. Therefore, $d_{n}(x) I=0$, which indicates that $d_{n}(x) \in t_{\tau}(M)$ and we have (3).

(3) $\Rightarrow(2)$. If $n=0$, then (3) $\Rightarrow(2)$ is trivial since we can let $I=(0: x)$. Now suppose that (3) $\Rightarrow(2)$ for every integer $k, 0 \leq k<n$, and let (3) hold for $n$. If $d: M \rightarrow M$ is a derivation of order $n$ and $x \in t_{\tau}(M)$, then since (3) holds for $n$, we have $d_{i}(x) \in t_{\tau}(M)$ for $i=0,1, \ldots, n$. Since $\left\{d_{i}: M \rightarrow M\right\}_{i=0}^{n-1}$ is a derivation on $M$ of order $n-1$, the induction hypothesis gives $I^{\prime} \in \mathscr{F}_{\tau}$ such that $\delta_{i}\left(I^{\prime}\right) \subseteq\left(0: d_{n-1-i}(x)\right)$ for $i=0,1, \ldots, n-1$. If $a \in I=$ $I^{\prime} \cap\left(0: d_{n}(x)\right) \in \mathscr{F}_{\tau}$, then

$$
d_{n}(x a)=d_{n}(x) a=d_{n-1}(x) \delta_{1}(a)=d_{n-2}(x) \delta_{2}(a)=\cdots=d_{1}(x) \delta_{n-1}(a)=0,
$$


SO

$$
d_{n}(x a)=d_{n}(x) a+d_{n-1}(x) \delta_{1}(a)+\cdots+d_{1}(x) \delta_{n-1}(a)+x \delta_{n}(a)
$$

becomes

$$
x \delta_{n}(a)=0 .
$$

Hence $\delta_{n}(I) \subseteq(0: x)$, so $\delta_{i}(I) \subseteq\left(0: d_{n-i}(x)\right)$ for $i=0,1, \ldots, n$.

$(2) \Rightarrow(1)$. It is obvious that $(2) \Rightarrow(1)$ when $n=0$, so suppose that $(2) \Rightarrow(1)$ for every integer $k$ such that $0 \leq k<n$. If $K \in \mathscr{F}_{\tau}$, then $R / K$ is $\tau$-torsion, so let $d=\left\{d_{i}: R / K \rightarrow R / K\right\}_{i=0}^{k}$, be a derivation on $R / K$ of order $k$ for $k=0,1, \ldots, n$. Since (2) holds for $k=0,1, \ldots, n-1$, for each such $k$ and each $x+K \in R / K$ there is an $I_{k, x} \in \mathscr{F}_{\tau}$ such that $\delta_{i}\left(I_{k, x}\right) \subseteq\left(0: d_{k-i}(x+\right.$ $K)$ ) for $i=0,1, \ldots, k$. In particular, for each $x+K \in R / K$, we have $\delta_{k}\left(I_{k, x}\right) \subseteq(0: x+K)$ for $k=0,1, \ldots, n-1$. If $a \in I_{x}=\left[\cap_{k=0}^{n-1} I_{k, x}\right] \cap\left(0: d_{n}(x+K)\right) \in \mathscr{F}_{\tau}$, then

$$
0=d_{n}((x+K) a)=d_{n}(x+K) a+d_{n-1}(x+K) \delta_{1}(a)+\cdots+(x+K) \delta_{n}(a)=(x+K) \delta_{n}(a) .
$$

Hence $\delta_{k}\left(I_{x}\right) \subseteq(0: x+K)$ for $k=0,1, \ldots, n$. If $x=1$, this gives $\delta_{k}\left(I_{1}\right) \subseteq(0: 1+K)=K$ for each $k$, so $\mathscr{F}_{\tau}$ is a differential filter of order $n$.

\section{Higher derivations and modules of quotients}

If $\tau$ is a torsion theory on $\operatorname{Mod}_{R}$, then an $R$-module $Q_{\tau}(M)$ together with an $R$ homomorphism $\varphi: M \rightarrow Q_{\tau}(M)$ is said to be a localization of $M$ at $\tau$ provided that $\operatorname{ker} \varphi$ and coker $\varphi$ are $\tau$-torsion and $Q_{\tau}(M)$ is $\tau$-injective and $\tau$-torsion-free. An $R$-module $M$ is said to be $\tau$-injective if $\operatorname{Hom}_{R}(-, M)=0$ preserves short exact sequences $0 \rightarrow N_{1} \rightarrow N \rightarrow$ $N_{2} \rightarrow 0$, where $N_{2}$ is a $\tau$-torsion module. The module $Q_{\tau}(M)$, called the module of quotients of $M$, is unique up to isomorphism whenever it can be shown to exist. Ohtake [14] has shown that a localization $\varphi: M \rightarrow Q_{\tau}(M)$ exists for every $R$-module $M$ if and only if the torsion theory is hereditary. It is well known that if $\tau$ is hereditary, then we can set $Q_{\tau}(M)=E_{\tau}\left(M / t_{\tau}(M)\right)$, where $E_{\tau}\left(M / t_{\tau}(M)\right)$ is the $\tau$-injective envelope of $M / t_{\tau}(M)[4,10]$. If $\eta: M \rightarrow M / t_{\tau}(M)$ is the natural surjection and $\mu: M / t_{\tau}(M) \rightarrow Q_{\tau}(M)$ is the canonical injection, then $\varphi=\mu \eta$.

If $d: M \rightarrow M$ is a derivation of order $n$, then we say that $d$ can be extended to a derivation $d^{\tau}: Q_{\tau}(M) \rightarrow Q_{\tau}(M)$ of order $n$ if the diagram

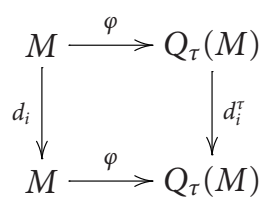

is commutative for $i=0,1, \ldots, n$. We can now show that a derivation $d: M \rightarrow M$ of order $n$ has a unique extension to a derivation $d^{\tau}: Q_{\tau}(M) \rightarrow Q_{\tau}(M)$ of order $n$ for every $R$-module $M$ if and only if $\tau$ is a differential torsion theory of order $n$. This result is established by the following lemma and proposition. 
Lemma 4.1. Let $\tau$ be a differential torsion theory of order $n$ on $\operatorname{Mod}_{R}$. If a derivation $d$ : $M \rightarrow M$ of order $n$ can be extended to a derivation $d^{\tau}: Q_{\tau}(M) \rightarrow Q_{\tau}(M)$ of order $n$, then $d^{\tau}$ is unique.

Proof. Let $\mathscr{F}_{\tau}$ be the differential filter of order $n$ corresponding to $\tau$ and suppose that $d^{\tau}$ and $\bar{d}^{\tau}$ are derivations of order $n$ that extend $d$ to $Q_{\tau}(M)$. Then for $k, 0 \leq k \leq n, d^{\tau}, \bar{d}^{\tau}$, and $d$ produce derivations $d^{* \tau}, \bar{d}^{* \tau}$, and $d^{*}$ of order $k$ and $d^{* \tau}$ and $\bar{d}^{* \tau}$ lift $d^{*}$. Since $\mathscr{F}_{\tau}$ is a differential filter of order $k$, if $x \in Q_{\tau}(M)$, then by Lemma 3.5 there are $I, I^{\prime} \in \mathscr{F}_{\tau}$ such that $\delta_{i}(I) \subseteq\left(0: d_{k-i}^{\tau}(x)\right)$ and $\delta_{i}\left(I^{\prime}\right) \subseteq\left(0: \bar{d}_{k-i}(x)\right)$ for $i=0,1, \ldots, k$. If $a \in K=I \cap I^{\prime} \in \mathscr{F}_{\tau}$, then for each $k$ we see that

$$
\begin{aligned}
0= & \left(d_{k}^{\tau}-\bar{d}_{k}^{\tau}\right)(x a) \\
= & d_{k}^{\tau}(x) a+d_{k-1}^{\tau}(x) \delta_{1}(a)+\cdots+x \delta_{k}(a) \\
& -\bar{d}_{k}^{\tau}(x) a-\bar{d}_{k-1}^{\tau}(x) \delta_{1}(a)-\cdots-x \delta_{k}(a) \\
= & {\left[d_{k}^{\tau}(x)-\bar{d}_{k}^{\tau}(x)\right] a+\left[d_{k-1}^{\tau}(x)-\bar{d}_{k-1}^{\tau}(x)\right] \delta_{1}(a) } \\
& +\cdots+\left[d_{1}^{\tau}(x)-\bar{d}_{1}^{\tau}(x)\right] \delta_{i-1}(a)+[x-x] \delta_{k}(a) \\
= & {\left[d_{k}^{\tau}(x)-\bar{d}_{k}(x)\right] a . }
\end{aligned}
$$

Hence, $\left[d_{k}^{\tau}(x)-\bar{d}_{k}^{\tau}(x)\right] K=0$, so $d_{k}^{\tau}(x)-\bar{d}_{k}^{\tau}(x) \in t_{\tau}\left(Q_{\tau}(M)\right)=0$. Therefore $d_{k}^{\tau}=\bar{d}_{k}^{\tau}$ for $k=0,1, \ldots, n$ and so $d^{\tau}=\bar{d}^{\tau}$.

We can now establish the main result of this section.

PROpOsition 4.2. Let $\tau$ be a hereditary torsion theory on $\operatorname{Mod}_{R}$. Then for every $R$-module $M$, each derivation $d: M \rightarrow M$ of order $n$ can be extended uniquely to a derivation $d^{\tau}$ : $Q_{\tau}(M) \rightarrow Q_{\tau}(M)$ of order $n$ if and only if $\tau$ is a differential torsion theory of order $n$.

Proof. Suppose that $d: M \rightarrow M$ is a derivation of order $n$. If $\tau$ is a differential torsion theory of order $n$, then $\mathscr{F}_{\tau}$ is a differential filter of order $n$, so it follows from Lemma 3.5 that $d_{i}\left(t_{\tau}(M)\right) \subseteq t_{\tau}(M)$ for $i=0,1, \ldots, n$. Hence $d$ can be extended to a derivation $d^{\tau}$ : $Q_{\tau}(M) \rightarrow Q_{\tau}(M)$ of order $n$ since Rim proved in [16] that such an extension exists when $d_{i}\left(t_{\tau}(M)\right) \subseteq t_{\tau}(M)$ for $i=0,1, \ldots, n$. Uniqueness follows from Lemma 4.1.

Conversely, suppose that every derivation $d: M \rightarrow M$ of order $n$ can be extended uniquely to a derivation $d^{\tau}: Q_{\tau}(M) \rightarrow Q_{\tau}(M)$ of order $n$. From the commutative diagram

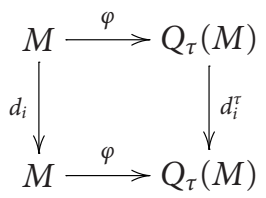

we see that $\varphi d_{i}=d_{i}^{\tau} \varphi$ for $i=0,1, \ldots, n$. So if $x \in t_{\tau}(M)=\operatorname{ker} \varphi$, then $\varphi d_{i}(x)=0$ for each $i$. This gives $d_{i}(x) \in \operatorname{ker} \varphi=t_{\tau}(M)$ and so we have $d_{i}\left(t_{\tau}(M)\right) \subseteq t_{\tau}(M)$ for $i=0,1, \ldots, n$. Calling on Lemma 3.5 again, we see that $\tau$ is a differential torsion theory of order $n$.

Corollary 4.3 [5, Proposition 2.3]. If $\tau$ is a hereditary torsion theory on $\operatorname{Mod}_{R}$, then for every $R$-module $M$, each derivation $d: M \rightarrow M$ can be extended uniquely to a derivation 
$d^{\tau}: Q_{\tau}(M) \rightarrow Q_{\tau}(M)$ if and only if $\tau$ is a differential torsion theory. In particular, $\tau$ is a differential torsion theory, then the derivation $\delta: R \rightarrow R$ extends uniquely to a derivation $\delta^{\tau}: Q_{\tau}(R) \rightarrow Q_{\tau}(R)$ defined on the ring of the quotients of $R$.

Proof. The first part of the corollary is clear if we consider $d: M \rightarrow M$ to be derivation of order 1 . Now let $M=R$ and apply this result to the derivation $\delta: R \rightarrow R$ to prove the second part of the corollary.

One consequence of Proposition 4.2 is that for a hereditary torsion theory $\tau$ on $\operatorname{Mod}_{R}$, the right ideals of the filter $\mathscr{F}_{\tau}$ form a test set for determining if derivations on $M$ of order $n$ can be extended uniquely to derivations on $Q_{\tau}(M)$ of order $n$.

\section{Higher derivations and modules of coquotients}

We now show that a result similar to Proposition 4.2 holds for colocalizations of modules whenever they universally exist. Colocalizations have been investigated under various approaches by several authors, for example, see $[3,6,13]$.

An $R$-module $C_{\tau}(M)$ together with an $R$-linear mapping $\varphi: C_{\tau}(M) \rightarrow M$ is said to be a colocalization of $M$ at $\tau$ provided that $\operatorname{ker} \varphi$ and coker $\varphi$ are $\tau$-torsion-free and $C_{\tau}(M)$ is $\tau$-torsion and $\tau$-projective. We call $C_{\tau}(M)$ the module of coquotients of $M$. We point out that $\tau$ is not assumed to be hereditary. When this is the case, a nonzero submodule of a $\tau$-torsion module can be $\tau$-torsion-free, a condition that is only possible for the zero submodule when $\tau$ is hereditary.

An $R$-module $M$ is $\tau$-projective if $\operatorname{Hom}_{R}(M,-)$ preserves short exact sequences $0 \rightarrow$ $N_{1} \rightarrow N \rightarrow N_{2} \rightarrow 0$, where $N_{1}$ is a $\tau$-torsion-free module. Ohtake was also able to show in [14] that a torsion theory $\tau$ is cohereditary if and only if every $R$-module $M$ has a colocalization at $\tau$. If $\varphi: C_{\tau}(M) \rightarrow M$ is a colocalization of $M$ at $\tau$, then there is an $R$ epimorphism $\pi: C_{\tau}(M) \rightarrow t_{\tau}(M)$ such that if $\mu: t_{\tau}(M) \rightarrow M$ is the canonical injection, then $\varphi=\mu \pi$. Furthermore, a module of coquotients is unique up to isomorphism whenever it can be shown to exist.

If $d: M \rightarrow M$ is a derivation of order $n$, then we say that $d$ can be lifted to a derivation $d^{\tau}: C_{\tau}(M) \rightarrow C_{\tau}(M)$ of order $n$ if the diagram

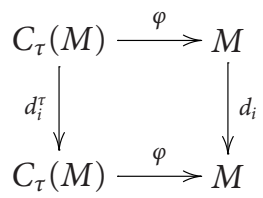

is commutative for $i=0,1, \ldots, n$. We will now show that such liftings are always possible at a TTF theory $\tau$ on $\operatorname{Mod}_{R}$.

When $\tau=(\mathrm{T}, \mathrm{F})$ is cohereditary, the class $\mathrm{F}$ of $\tau$ is both a torsion and a torsion-free class, and the class $\mathrm{F}$ generates a hereditary torsion theory $\sigma=(\mathrm{F}, \mathrm{D})$ on $\operatorname{Mod}_{R}$. The pair $(\tau, \sigma)$ is often referred to as a TTF theory. Jans has shown in [11] that there is a oneto-one correspondence between TTF theories and idempotent ideals $I$ of $R$. If $(\tau, \sigma)$ is a TTF theory with corresponding idempotent ideal $I$, then the filter determined by $\sigma$ is 
given by $\mathscr{F}_{\sigma}=\{K \subseteq R \mid K \supseteq I, K$ a right ideal of $R\}$. In this setting, $t_{\tau}(R)=I$ and $t_{\tau}(M)=$ $M I$ for each $R$-module $M$. We have seen in Example 3.3 that $\sigma$ is a higher differential torsion theory although this condition on $\sigma$ is not a factor in lifting higher derivations $d: M \rightarrow M$ to higher derivations $d^{\tau}: C_{\tau}(M) \rightarrow C_{\tau}(M)$. Sato has shown in [17] that if $\tau$ is cohereditary, then $I \otimes_{R} I \stackrel{\pi}{\rightarrow} I \stackrel{\mu}{\rightarrow} R$ is a colocalization of $R$, where the map $\pi: I \otimes_{R} I \rightarrow I$ is given by $\sum_{i=1}^{n}\left(a_{i} \otimes b_{i}\right) \mapsto \sum_{i=1}^{n} a_{i} b_{i}$. Furthermore, $I \otimes_{R} I$ is a ring, possibly without an identity, and an $(R, R)$-bimodule. Sato also shows in [17] that $M \otimes_{R} I \otimes_{R} I \stackrel{\pi}{\rightarrow} M I \stackrel{\mu}{\rightarrow} M$ is a colocalization of $M$ at $\tau$, where the map $\pi: M \otimes_{R} I \otimes_{R} I \rightarrow M I$ is given by $\sum_{i-1}^{n}\left(x_{i} \otimes a_{i} \otimes\right.$ $\left.b_{i}\right) \mapsto \sum_{i=1}^{n} x_{i} a_{i} b_{i}$. Since $I$ is an idempotent ideal, $\delta_{i}(I) \subseteq I$ for $i=0,1, \ldots, n$ and it follows that each derivation $d: M \rightarrow M$ of order $n$ is such that $d_{i}(M I) \subseteq M I$ for $i=0,1, \ldots, n$. Hence, $d$ restricted to $M I$ produces a derivation $d: M I \rightarrow M I$ of order $n$ that will also be denoted by $d$.

We now need the following lemma.

Lemma 5.1. If $I$ is an idempotent ideal of $R$ and $d: M \rightarrow M$ is a derivation of order $n$, then the map $\rho_{i}: M \times I \times I \rightarrow M \otimes_{R} I \otimes_{R} I$ given by

$$
\rho_{i}(x, a, b)=\sum_{j=0}^{i} d_{i-j}(x) \otimes\left[\sum_{k=0}^{j} \delta_{j-k}(a) \otimes \delta_{k}(b)\right]
$$

is $R$-balanced for $i=0,1, \ldots, n$. That is, $\rho_{i}$ is additive in each variable and such that $\rho_{i}(x r, a, b)$ $=\rho_{i}(x, r a, b)$ and $\rho_{i}(x, a r, b)=\rho_{i}(x, a, r b)$ for $(x, a, b) \in M \times I \times I$ and $r \in R$.

Proof. We show $\rho_{i}(x r, a, b)=\rho_{i}(x, r a, b)$. The proof that $\rho_{i}(x, a r, b)=\rho_{i}(x, a, r b)$ is similar and so is omitted. Expanding $\sum_{j=0}^{i} d_{i-j}(x r) \otimes\left[\sum_{k=0}^{j} \delta_{j-k}(a) \otimes \delta_{k}(b)\right]$ by the first summation, we have

$$
\begin{aligned}
d_{i}(x r) & \otimes\left[\sum_{k=0}^{0} \delta_{0-k}(a) \otimes \delta_{k}(b)\right]+d_{i-1}(x r) \otimes\left[\sum_{k=0}^{1} \delta_{1-k}(a) \otimes \delta_{k}(b)\right] \\
& +d_{i-2}(x r) \otimes\left[\sum_{k=0}^{2} \delta_{2-k}(a) \otimes \delta_{k}(b)\right]+\cdots+x r \otimes\left[\sum_{k=0}^{i} \delta_{i-k}(a) \otimes \delta_{k}(b)\right] .
\end{aligned}
$$

Using (5.3) and the definition of $d_{i-j}$ for $j=0,1, \ldots, n$, we get

$$
\begin{aligned}
{\left[\sum_{s=0}^{i} d_{i-s}(x) \delta_{s}(r)\right] \otimes\left[\sum_{k=0}^{0} \delta_{0-k}(a) \otimes \delta_{k}(b)\right] } \\
+\left[\sum_{s=0}^{i-1} d_{i-1-s}(x) \delta_{s}(r)\right] \otimes\left[\sum_{k=0}^{1} \delta_{1-k}(a) \otimes \delta_{k}(b)\right] \\
+\left[\sum_{s=0}^{i-2} d_{i-2-s}(x) \delta_{s}(r)\right] \otimes\left[\sum_{k=0}^{2} \delta_{2-k}(a) \otimes \delta_{k}(b)\right] \\
+\cdots+x r \otimes\left[\sum_{k=0}^{i} \delta_{i-k}(a) \otimes \delta_{k}(b)\right]
\end{aligned}
$$


2382 Higher derivations on rings and modules

which, by shifting subscripts, can be written as

$$
\begin{aligned}
& {\left[\sum_{s=0}^{i} d_{i-s}(x) \delta_{s}(r)\right] \otimes\left[\sum_{k=0}^{0} \delta_{0-k}(a) \otimes \delta_{k}(b)\right]} \\
& +\left[\sum_{s=1}^{i} d_{i-s}(x) \delta_{s-1}(r)\right] \otimes\left[\sum_{k=0}^{1} \delta_{1-k}(a) \otimes \delta_{k}(b)\right] \\
& +\left[\sum_{s=2}^{i} d_{i-s}(x) \delta_{s-2}(r)\right] \otimes\left[\sum_{k=0}^{2} \delta_{2-k}(a) \otimes \delta_{k}(b)\right] \\
& +\cdots+x r \otimes\left[\sum_{k=0}^{i} \delta_{i-k}(a) \otimes \delta_{k}(b)\right] .
\end{aligned}
$$

Using properties of tensor products, (5.5) becomes

$$
\begin{aligned}
& {\left[\sum_{s=0}^{i} d_{i-s}(x)\right] \otimes\left[\sum_{k=0}^{0} \delta_{s}(r) \delta_{0-k}(a) \otimes \delta_{k}(b)\right]} \\
& +\left[\sum_{s=1}^{i} d_{i-s}(x)\right] \otimes\left[\sum_{k=0}^{1} \delta_{s-1}(r) \delta_{1-k}(a) \otimes \delta_{k}(b)\right] \\
& +\left[\sum_{s=2}^{i} d_{i-s}(x)\right] \otimes\left[\sum_{k=0}^{2} \delta_{s-2}(r) \delta_{2-k}(a) \otimes \delta_{k}(b)\right] \\
& +\cdots+x \otimes\left[\sum_{k=0}^{i} r \delta_{i-k}(a) \otimes \delta_{k}(b)\right] .
\end{aligned}
$$

We now use (5.6) to compute the $(i-t)$ th term, where $0 \leq t \leq i$. Each summand $\left[\sum_{s=u}^{i} d_{i-s}(x)\right] \otimes\left[\sum_{k=0}^{u} \delta_{s-u}(r) \delta_{u-k}(a) \otimes \delta_{k}(b)\right]$ in (5.6) contains an $(i-t)$ th term until $t>u$. These terms are

$$
\begin{aligned}
d_{i-t}(x) \otimes\left[\sum_{k=0}^{0} \delta_{t}(r) \delta_{0-k}(a) \otimes \delta_{k}(b)\right] \\
+d_{i-t}(x) \otimes\left[\sum_{k=0}^{1} \delta_{t-1}(r) \delta_{1-k}(a) \otimes \delta_{k}(b)\right] \\
+d_{i-t}(x) \otimes\left[\sum_{k=0}^{2} \delta_{t-2}(r) \delta_{2-k}(a) \otimes \delta_{k}(b)\right] \\
+\cdots+d_{i-t}(x) \otimes\left[\sum_{k=0}^{t} r \delta_{t-k}(a) \otimes \delta_{k}(b)\right] .
\end{aligned}
$$


Next use (5.7) to compute and group the terms corresponding to $k=0, k=1$, and so on until $k=t$. This produces

$$
\begin{aligned}
d_{i-t}(x) & \otimes\left[\delta_{t}(r) a+\delta_{t-1}(r) \delta_{1}(a)+\delta_{t-2}(r) \delta_{2}(a)+\cdots+r \delta_{t}(a)\right] \otimes b \\
+ & d_{i-t}(x) \otimes\left[\delta_{t-1}(r) a+\delta_{t-2}(r) \delta_{1}(a)+\cdots+r \delta_{t-1}(a)\right] \otimes \delta_{1}(b) \\
+ & d_{i-t}(x) \otimes\left[\delta_{t-2}(r) a+\delta_{t-3}(r) \delta_{1}(a)+\cdots+r \delta_{t-2}(a)\right] \otimes \delta_{2}(b) \\
& +\cdots+d_{i-t}(x) \otimes r a \otimes \delta_{t}(b)
\end{aligned}
$$

which reduces to

$$
\begin{aligned}
& d_{i-t}(x) \otimes \delta_{t}(r a) \otimes b+d_{i-t}(x) \otimes \delta_{t-1}(r a) \otimes \delta_{1}(b) \\
& +d_{i-t}(x) \otimes \delta_{t-2}(r a) \otimes \delta_{2}(b)+\cdots+d_{i-t}(x) \otimes r a \otimes \delta_{t}(b) .
\end{aligned}
$$

The last expression (5.9) sums to

$$
d_{i-t}(x) \otimes\left[\sum_{k=0}^{t} \delta_{t-k}(r a) \otimes \delta_{k}(b)\right]
$$

and since this holds for any $t$ with $0 \leq t \leq i$, it follows that

$$
\sum_{j=0}^{i} d_{i-j}(x r) \otimes\left[\sum_{k=0}^{j} \delta_{j-k}(a) \otimes \delta_{k}(b)\right]=\sum_{t=0}^{i} d_{i-t}(x) \otimes\left[\sum_{k=0}^{t} \delta_{t-k}(r a) \otimes \delta_{k}(b)\right] .
$$

Hence $\rho_{i}(x r, a, b)=\rho_{i}(x, r a, b)$.

Lemma 5.2. If $\tau$ is a cohereditary torsion theory on $\operatorname{Mod}_{R}$ and a derivation $d: M \rightarrow M$ of order $n$ lifts to a derivation $d^{\tau}: C_{\tau}(M) \rightarrow C_{\tau}(M)$ of order $n$, then $d^{\tau}$ is unique.

Proof. If $n=0$, then $d_{0}$ is the identity map on $M$, so suppose that $d^{\tau}, \bar{d}^{\tau}: C_{\tau}(M) \rightarrow$ $C_{\tau}(M)$, both of order 0 , lift $d$ to $C_{\tau}(M)$. Then by definition $d_{0}^{\tau}=\bar{d}_{0}^{\tau}=i d_{C_{\tau}(M)}$, so $d^{\tau}=\bar{d}^{\tau}$. Make the induction hypothesis that each derivation $d: M \rightarrow M$ of order $k, 0 \leq k<n$, lifts uniquely to $C_{\tau}(M)$ by a derivation of order $k$. If $d: M \rightarrow M$ is a derivation of order $n$ and $d^{\tau}, \bar{d}^{\tau}: C_{\tau}(M) \rightarrow C_{\tau}(M)$ are derivations of order $n$ that lift $d$, then $d, d^{\tau}$, and $\bar{d}^{\tau}$ produce derivations $d^{*}, d^{* \tau}$, and $\bar{d}^{* \tau}$ of order $n-1$ and $d^{* \tau}$ and $\bar{d}^{* \tau}$ lift $d^{*}$. Hence the induction hypothesis gives $d_{i}^{\tau}=\bar{d}_{i}^{\tau}$ for $i=0,1, \ldots, n-1$. If $x \in C_{\tau}(M)$ and $r \in R$, then $\left(d^{\tau}-\bar{d}^{\tau}\right)(x r)=\sum_{j=0}^{n}\left(d_{n-j}^{\tau}-\bar{d}_{n-j}^{\tau}\right)(x) \delta_{j}(r)$, so $\left(d^{\tau}-\bar{d}^{\tau}\right)(x r)=\left(d_{n}^{\tau}-\bar{d}_{n}^{\tau}\right)(x) r$. Thus $d^{\tau}-\bar{d}^{\tau}$ is an $R$-linear mapping which means that $\operatorname{Im}\left(d^{\tau}-\bar{d}^{\tau}\right)$ is $\tau$-torsion. Since $d_{n}^{\tau}$ and $\bar{d}_{n}^{\tau}$ render the diagram

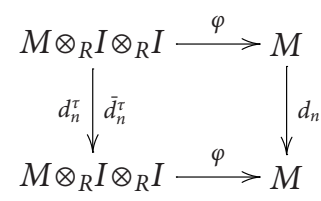

commutative, it follows that $\operatorname{Im}\left(d^{\tau}-\bar{d}^{\tau}\right) \subseteq \operatorname{ker} \varphi$. Hence $\operatorname{Im}\left(d^{\tau}-\bar{d}^{\tau}\right)$ is also $\tau$-torsionfree and so $\operatorname{Im}\left(d^{\tau}-\bar{d}^{\tau}\right)=0$. Therefore $d^{\tau}=\bar{d}^{\tau}$. 
Proposition 5.3. If $\tau$ is a cohereditary torsion theory on $\operatorname{Mod}_{R}$, then every derivation $d$ : $M \rightarrow M$ of order $n$ lifts uniquely to a derivation $d^{\tau}: C_{\tau}(M) \rightarrow C_{\tau}(M)$ of order $n$.

Proof. If $\tau=(\mathrm{T}, \mathrm{F})$ and $\sigma=(\mathrm{F}, \mathrm{D})$ is the torsion theory generated by $\mathrm{F}$, let $I$ be the idempotent ideal corresponding to the TTF theory $(\tau, \sigma)$. If $d: M \rightarrow M$ is a derivation of order $n$, then, for each $i$, we have a commutative diagram

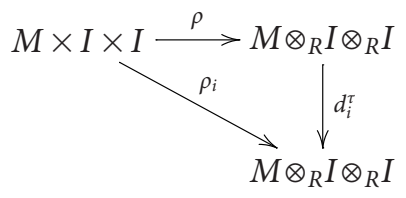

where $\rho: M \times I \times I \rightarrow M \otimes_{R} I \otimes_{R} I$ is the canonical $R$-balanced map given by $\rho(x, a, b)=$ $x \otimes a \otimes b, \rho_{i}$ is the $R$-balanced map of Lemma 5.1, and $d_{i}^{\tau}$ is the group homomorphism produced by the tensor product $M \otimes_{R} I \otimes_{R} I$. It follows that

$$
d_{i}^{\tau}(x \otimes a \otimes b)=\rho_{i}(x, a, b)=\sum_{j=0}^{i} d_{i-j}(x) \otimes\left[\sum_{k=0}^{j} \delta_{j-k}(a) \otimes \delta_{k}(b)\right]
$$

for each $(x, a, b) \in M \times I \times I$, so, for each $i$, consider the commutative diagram

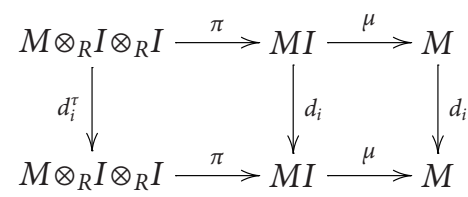

Since $\varphi=\mu \pi$, where $\pi: M \otimes_{R} I \otimes_{R} I \rightarrow M I$ is such that $\pi\left(\sum_{i=1}^{n}\left(x_{i} \otimes a_{i} \otimes b_{i}\right)\right)=\sum_{i=1}^{n} x_{i} a_{i} b_{i}$ and $\mu: M I \rightarrow M$ is the canonical injection, we see that $\varphi\left(\sum_{i=1}^{n}\left(x_{i} \otimes a_{i} \otimes b_{i}\right)\right)=\sum_{i=1}^{n} x_{i} a_{i} b_{i}$ for each $\sum_{i=1}^{n}\left(x_{i} \otimes a_{i} \otimes b_{i}\right) \in M \otimes_{R} I \otimes_{R} I$. Now let $x \otimes a \otimes b$ be a generator of $M \otimes_{R} I \otimes_{R} I$. Then

$$
\begin{aligned}
\varphi d_{i}^{\tau}(x \otimes a \otimes b) & =\varphi \rho_{i}(x, a, b) \\
& =\varphi\left(\sum_{j=0}^{i} d_{i-j}(x) \otimes\left[\sum_{k=0}^{j} \delta_{j-k}(a) \otimes \delta_{k}(b)\right]\right) \\
& =\sum_{j=0}^{i} d_{i-j}(x)\left[\sum_{k=0}^{j} \delta_{j-k}(a) \delta_{k}(b)\right] \\
& =\sum_{j=0}^{i} d_{i-j}(x) \delta_{j}(a b) \\
& =d_{i}(x a b) \\
& =d_{i} \varphi(x \otimes a \otimes b) .
\end{aligned}
$$


Since $\varphi d_{i}^{\tau}$ and $d_{i} \varphi$ are additive functions, this suffices to show that $\varphi d_{i}^{\tau}=d_{i} \varphi$, so the diagram

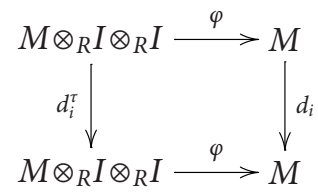

is commutative for each $i$. Finally, to show that $d^{\tau}$ is a derivation of order $n$, we need to show for $i=0,1, \ldots, n$ that

$$
d_{i}^{\tau}((x \otimes a \otimes b) r)=\sum_{j=0}^{i} d_{i-j}(x \otimes a \otimes b) \delta_{j}(r)
$$

for each generator $x \otimes a \otimes b \in M \otimes_{R} I \otimes_{R} I$ and $r \in R$. Now

$$
\begin{aligned}
\sum_{j=0}^{i} d_{i-j}(x \otimes a \otimes b) \delta_{j}(r) \\
=d_{i}^{\tau}(x \otimes a \otimes b) r+d_{i-1}^{\tau}(x \otimes a \otimes b) \delta_{1}(r) \\
\quad+d_{i-2}^{\tau}(x \otimes a \otimes b) \delta_{2}(r)+\cdots+(x \otimes a \otimes b) \delta_{i}(r)
\end{aligned}
$$

and the expression on the right of the equality in (5.19) gives

$$
\begin{aligned}
{\left[\sum_{j=0}^{i} d_{i-j}(x) \otimes\left[\sum_{k=0}^{j} \delta_{j-k}(a) \otimes \delta_{k}(b)\right]\right] r } \\
+\left[\sum_{j=0}^{i-1} d_{i-1-j}(x) \otimes\left[\sum_{k=0}^{j} \delta_{j-k}(a) \otimes \delta_{k}(b)\right]\right] \delta_{1}(r) \\
+\left[\sum_{j=0}^{i-2} d_{i-2-j}(x) \otimes\left[\sum_{k=0}^{j} \delta_{j-k}(a) \otimes \delta_{k}(b)\right]\right] \delta_{2}(r)+\cdots+(x \otimes a \otimes b) \delta_{i}(r) .
\end{aligned}
$$

Using properties of tensor products, (5.20) becomes

$$
\begin{aligned}
& \sum_{j=0}^{i} d_{i-j}(x) \otimes \delta_{j}(a b) \otimes r+\sum_{j=0}^{i-1} d_{i-1-j}(x) \otimes \delta_{j}(a b) \otimes \delta_{1}(r) \\
& \quad+\sum_{j=0}^{i-2} d_{i-2-j}(x) \otimes \delta_{j}(a b) \otimes \delta_{2}(r)+\cdots+\left(x \otimes a b \otimes \delta_{i}(r)\right) .
\end{aligned}
$$


2386 Higher derivations on rings and modules

Expanding (5.21) produces

$$
\begin{gathered}
d_{i}(x) \otimes a b \otimes r+d_{i-1}(x) \otimes \delta_{1}(a b) \otimes r+d_{i-2}(x) \otimes \delta_{2}(a b) \otimes r+\cdots \\
+d_{i-1}(x) \otimes a b \otimes \delta_{1}(r)+d_{i-2}(x) \otimes \delta_{1}(a b) \otimes \delta_{1}(r)+\cdots \\
+d_{i-2}(x) \otimes a b \otimes \delta_{2}(r)+\cdots+x \otimes \delta_{i}(a b) \otimes r \\
+x \otimes \delta_{i-1}(a b) \otimes \delta_{1}(r)+x \otimes \delta_{i-2}(a b) \otimes \delta_{2}(r) \\
\vdots \\
+x \otimes a b \otimes \delta_{i}(r) .
\end{gathered}
$$

By summing (5.22) down the columns, we have

$$
\begin{gathered}
d_{i}(x) \otimes a b \otimes r+d_{i-1}(x) \otimes \sum_{k=0}^{1} \delta_{1-k}(a b) \otimes \delta_{k}(r) \\
+d_{i-2}(x) \otimes \sum_{k=0}^{2} \delta_{2-k}(a b) \otimes \delta_{k}(r) \\
+\cdots+x \otimes \sum_{k=0}^{i} \delta_{i-k}(a b) \otimes \delta_{k}(r)
\end{gathered}
$$

which gives

$$
\begin{aligned}
\sum_{j=0}^{i} d_{i-j}(x) \otimes \sum_{k=0}^{n} \delta_{j-k}(a b) \otimes \delta_{k}(r) \\
=d_{i}^{\tau}(x \otimes a b \otimes r) \\
=d_{i}^{\tau}(x \otimes a \otimes b r) \\
=d_{i}^{\tau}((x \otimes a \otimes b) r) .
\end{aligned}
$$

Uniqueness follows from Lemma 5.2.

The proof of the first part of the corollary follows from the proposition by considering the derivation $d: M \rightarrow M$ to be a higher derivation of order 1 and the lifting of $\delta$ follows from this and the observation that $R \otimes_{R} I \otimes_{R} I \cong I \otimes_{R} I$ and $R I=I$.

\section{References}

[1] J. S. Alin and E. P. Armendariz, TTF-classes over perfect rings, J. Austral. Math. Soc. Ser. A 11 (1970), 499-503.

[2] F. W. Anderson and K. R. Fuller, Rings and Categories of Modules, Graduate Texts in Mathematics, vol. 13, Springer, Berlin, 1974.

[3] J. A. Beachy, Cotorsion radicals and projective modules, Bull. Austral. Math. Soc. 5 (1971), 241253.

[4] P. E. Bland, Topics in Torsion Theory, Mathematical Research, vol. 103, Wiley-VCH, Berlin, 1998.

[5] Differential torsion theory, to appear in J. Pure Appl. Algebra. 
[6] S. D. Bronn, Cotorsion theories, Pacific J. Math. 48 (1973), no. 2, 355-363.

[7] V. Dlab, A characterization of perfect rings, Pacific J. Math. 33 (1970), no. 1, 79-88.

[8] P. Gabriel, Des catégories abéliennes, Bull. Soc. Math. France 90 (1962), 323-448 (French).

[9] J. S. Golan, Extension of derivations to modules of quotients, Comm. Algebra 9 (1981), no. 3, 275-281.

[10] Torsion Theories, Pitman Monographs and Surveys in Pure and Applied Mathematics, vol. 29, Longman Scientific \& Technical, Harlow; John Wiley \& Sons, New York, 1986.

[11] J. P. Jans, Some aspects of torsion, Pacific J. Math. 15 (1965), no. 4, 1249-1259.

[12] T. Y. Lam, Lectures on Modules and Rings, Graduate Texts in Mathematics, vol. 189, Springer, New York, 1999.

[13] R. J. McMaster, Cotorsion theories and colocalization, Canad. J. Math. 27 (1975), no. 3, 618-628.

[14] K. Ohtake, Colocalization and localization, J. Pure Appl. Algebra 11 (1977/78), no. 1-3, 217241.

[15] P. Ribenboim, Higher order derivations of modules, Portugal. Math. 39 (1980), no. 1-4, 381-397 (1985).

[16] S. H. Rim, Extensions of higher anti-derivations to modules of quotients, J. Korean Math. Soc. 24 (1987), no. 1, 25-31.

[17] M. Sato, The concrete description of the colocalization, Proc. Japan Acad. 52 (1976), no. 9, 501504.

Paul E. Bland: Department of Mathematics, Eastern Kentucky University, Richmond, KY 40475, USA

E-mail address: paul.bland@eku.edu 


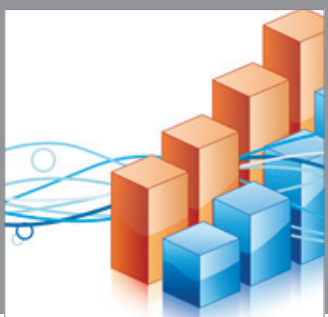

Advances in

Operations Research

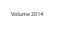

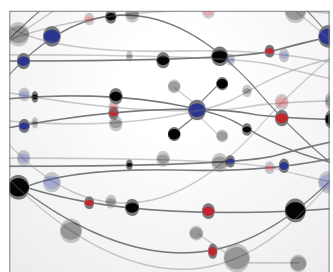

\section{The Scientific} World Journal
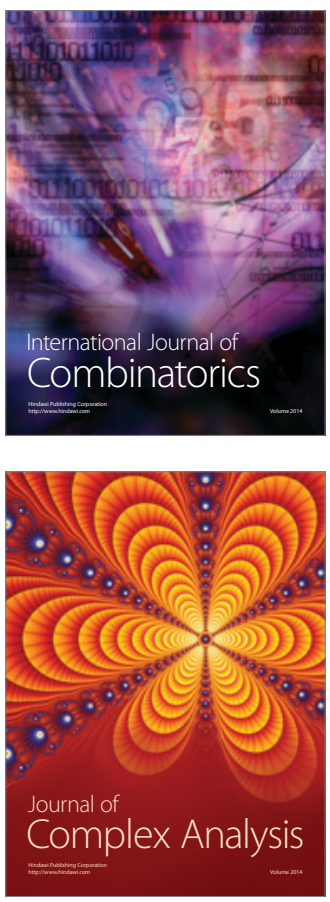

International Journal of

Mathematics and

Mathematical

Sciences
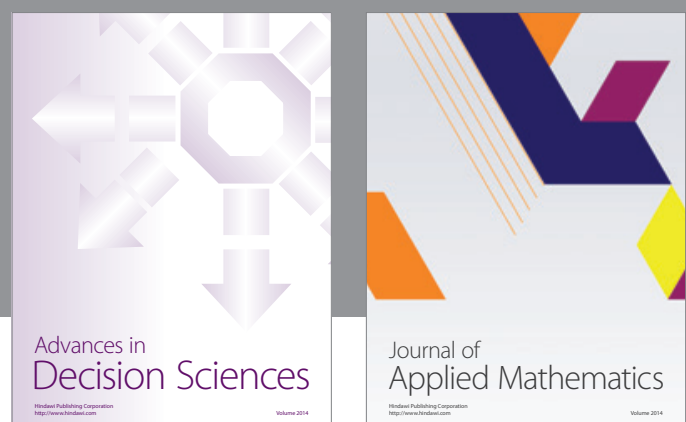

Journal of

Applied Mathematics
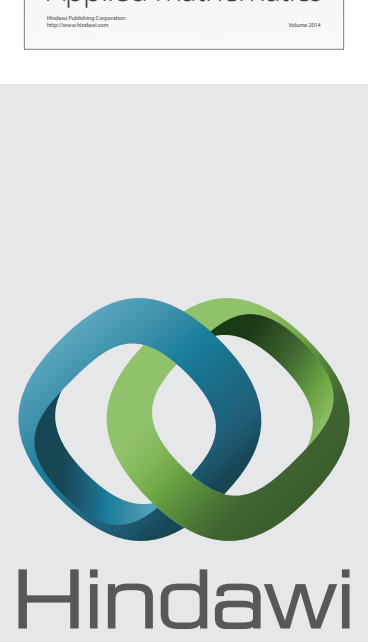

Submit your manuscripts at http://www.hindawi.com
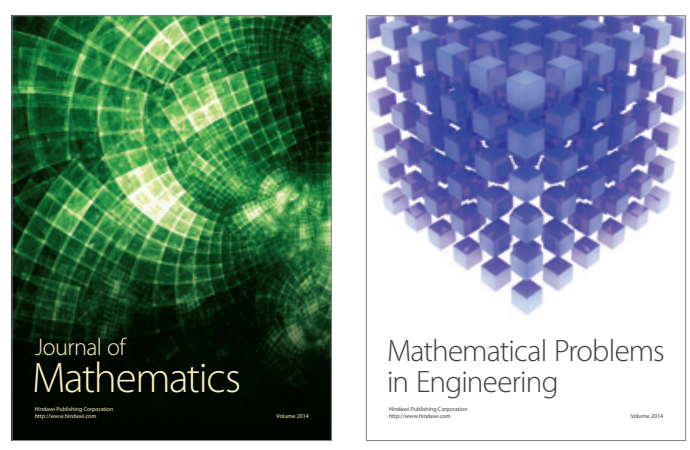

Mathematical Problems in Engineering
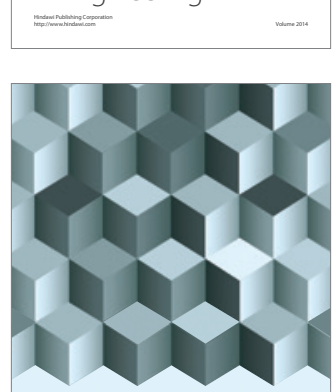

Journal of

Function Spaces
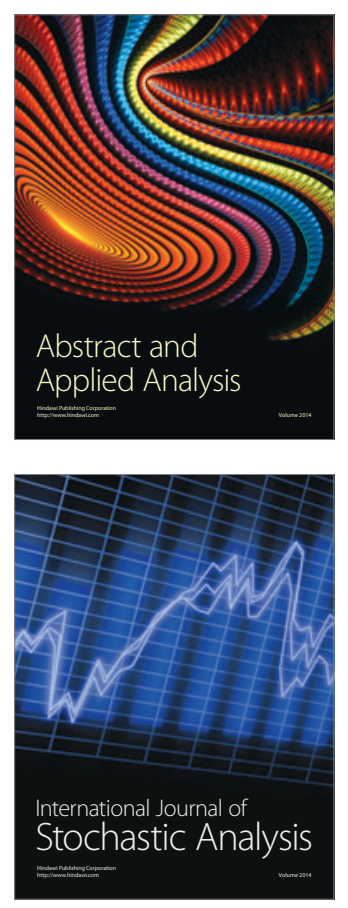

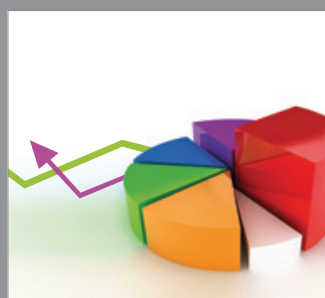

ournal of

Probability and Statistics

Promensencen
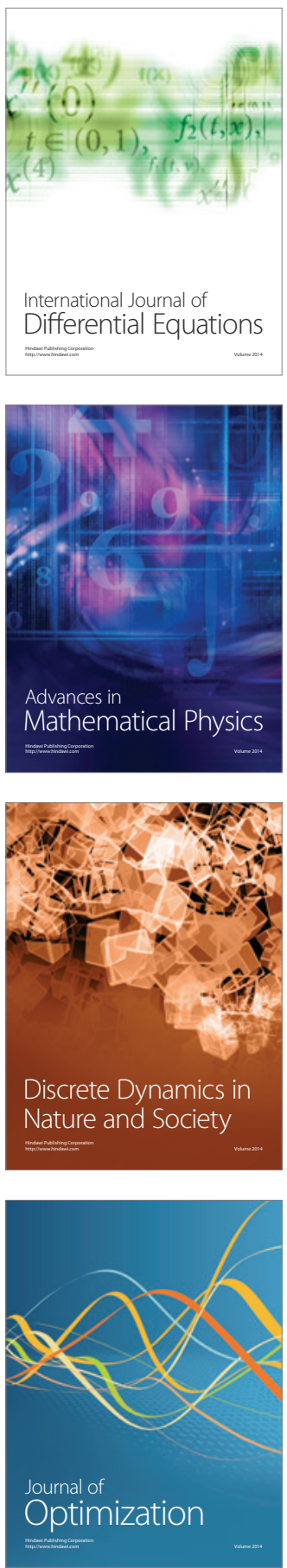